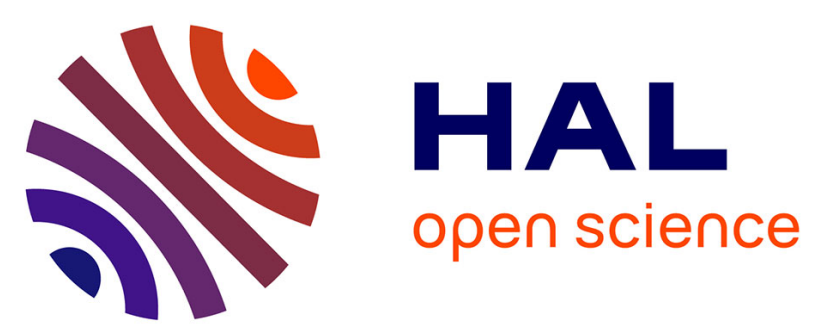

\title{
Development and application of a new unsteady far-field drag decomposition method
}

H. Toubin, D. Bailly

\section{To cite this version:}

H. Toubin, D. Bailly. Development and application of a new unsteady far-field drag decomposition method. AIAA AVIATION 2014, Jun 2014, ATLANTA, United States. hal-01069591

\section{HAL Id: hal-01069591 \\ https://hal-onera.archives-ouvertes.fr/hal-01069591}

Submitted on 29 Sep 2014

HAL is a multi-disciplinary open access archive for the deposit and dissemination of scientific research documents, whether they are published or not. The documents may come from teaching and research institutions in France or abroad, or from public or private research centers.
L'archive ouverte pluridisciplinaire HAL, est destinée au dépôt et à la diffusion de documents scientifiques de niveau recherche, publiés ou non, émanant des établissements d'enseignement et de recherche français ou étrangers, des laboratoires publics ou privés. 


\title{
Development and Application of a New Unsteady Far-Field Drag Decomposition Method
}

\author{
Hélène Toubin* and Didier Bailly ${ }^{\dagger}$ \\ Onera, The French Aerospace Lab, F92190 Meudon, France
}

\begin{abstract}
Far-field decomposition methods are among the most powerful means to accurately compute the forces on an aircraft. They allow distinguishing between the drag components associated with the various physical phenomena: shock waves, viscous interactions and liftinduced vortex, without being readily applicable to unsteady flows. Van der Vooren and Destarac have for example developed a powerful and reliable method used widely in the industry but restricted to steady flows. Gariépy has made a first attempt in generalizing it but his decomposition still holds terms that cannot be matched with physical phenomena. This paper presents another generalization to unsteady flows of Van der Vooren formulation. The proposed method relies on a strong theoretical background and allows the breakdown of drag into the three usual components only. This new unsteady formulation is applied to an OAT15A profile under buffeting condition, then to a NACA0012 profile at high angle of attack with natural vortex shedding, and finally to an oscillating profile. The results are analyzed and compared to the only other formulation available to break down the drag of unsteady configurations.
\end{abstract}

\section{Nomenclature}

$M \quad$ Mach number

Re Reynolds number

$\rho$ density

$\boldsymbol{q} \quad$ velocity vector

$u, v, w$ velocity components in an inertial reference frame

$p \quad$ static pressure

$p_{i} \quad$ stagnation pressure $p_{i}=p\left(1+\frac{\gamma-1}{2} M^{2}\right)^{\frac{\gamma}{\gamma-1}}$

$t \quad$ time

$\boldsymbol{\tau}$ deviatoric stress tensor

$\tau_{x} \quad$ longitudinal stress vector $\tau_{x}=\boldsymbol{\tau} \cdot \boldsymbol{i}$

$T$ temperature

$s \quad$ entropy

$h \quad$ enthalpy

$H \quad$ stagnation enthalpy $H=h+\frac{q^{2}}{2}$

$\mathrm{C}_{\mathrm{p}} \quad$ specific pressure heat

$\gamma \quad$ ratio of specific heats

r gas constant

$\alpha \quad$ angle of attack

$u_{i r r} \quad$ axial velocity under irreversible flow assumptions

$u_{i r r}^{*} \quad$ enthalpy-corrected axial velocity under irreversible flow assumptions

$u_{\text {rev }}$ axial velocity under reversible flow assumptions

$u_{\text {rev }}^{*} \quad$ enthalpy-corrected axial velocity under reversible flow assumptions

*PhD Student, Applied Aerodynamics Dept., Helene.Toubin@onera.fr.

${ }^{\dagger}$ Research Engineer, Applied Aerodynamics Dept., Didier.Bailly@onera.fr. 


$\begin{array}{ll}D_{p} & \text { pressure drag } \\ D_{f} & \text { friction drag } \\ D_{n f} & \text { near-field drag } \\ D_{v w} & \text { profile drag } \\ D_{w} & \text { wave drag } \\ D_{v} & \text { viscous drag } \\ D_{i} & \text { induced drag } \\ D_{m} & \text { motion drag } \\ D_{f f} & \text { far-field drag } \\ D_{s p} & \text { spurious drag } \\ \boldsymbol{n} & \text { normal vector pointing outside the flow domain } \\ \boldsymbol{i} & \text { freestream direction vector } \\ V & \text { control volume } \\ S_{a} & \text { surface of the body } \\ S_{e} & \text { outer surface of the fluid volume } \\ S_{d} & \text { downstream wake plane } \\ S_{w} & \text { surface for the integration of wave drag } \\ S_{v} & \text { surface for the integration of viscous drag } \\ V_{w} & \text { volume enclosed within } S_{w} \\ V_{w d} & \text { volume downstream of } V_{w} \\ V_{v} & \text { volume enclosed within } S_{v} \\ V_{d} & \text { complementary volume } V_{d}=V \backslash\left(V_{w} \cup V_{w d} \cup V_{v}\right) \\ S u b s c r i p t & \text { freestream state } \\ \infty & \end{array}$

\section{Introduction}

$\mathrm{T}$ HE reduction of the aircraft fuel consumption is tightly bound to the reduction of drag. Even a slight improvement on the drag can allow a non-negligible gain on the payload. Flow control, aerodynamic optimization or exploration of innovative configurations are three available means to enhance the aerodynamic performance. An accurate and reliable drag prediction method is therefore required. Complex unsteady configurations are in the meantime becoming more common and achievable with the computing resources. This context seems therefore the right timing for the development of an unsteady drag prediction tool. Examples of application would be rotors, helicopters, counter-rotating open rotors (CROR), flutter or vortex shedding downstream of a spoiler.

Far-field drag computation was first introduced by Betz. ${ }^{1}$ Instead of computing the forces on a body by integrating aerodynamic stresses on the skin (near-field method), one can equivalently analyze the aerodynamic phenomena which occur within the fluid surrounding the body. This analysis is richer and allows distinguishing between the drag components associated with the various physical phenomena: shock waves, viscous interactions in the boundary layer and in the wake, and lift-induced vorticity. However, no far-field method is for now able to successfully break down the drag of unsteady flows. Noca ${ }^{2}$ has carried out an experimental study of several far-field formulations based on $\mathrm{Wu}^{3}$ equations. Marongiu ${ }^{4}$ as well as $\mathrm{Xu}^{5}$ have applied similar methods to numerical unsteady flows, but like Noca, without achieving a physical breakdown. Gariépy ${ }^{6}$ has also made a first attempt in generalizing Van der Vooren and Destarac ${ }^{7}$ formulation, which has proven its robustness and accuracy for steady flows, but his decomposition still holds terms that cannot be matched with physical phenomena and which are designated unsteady drag.

This paper presents a generalization of Van der Vooren formulation to unsteady flows. It results in only viscous, wave, induced and spurious unsteady drag, and is valid for both URANS and DES simulations. This new unsteady formulation is compared to the only other unsteady drag decomposition method, that of Gariépy. Since Gariépy ${ }^{8}$ uses Méheut's expression for the axial velocity defect, ${ }^{9}$ both approaches are first tested on steady cases. The unsteady formulation is then applied to URANS computations: an OAT15A profile with buffeting, a NACA0012 profile at high angle of attack with natural vortex shedding, and a NACA0012 profile oscillating at low speed. 


\section{Theory}

In this section, the general equations which lead to the far-field formulation will be presented. The formulation will then be generalized to unsteady flows, leading to a new decomposition. Gariépy's formulation will also be analyzed and compared to our new formulation.

\section{A. General equations for drag computation}

The far-field theory consists in computing the aerodynamic force from the flow field analysis instead of the integration of the local stress on the body. The balance between the two approaches relies on the conservation of fluid momentum. It requires no further hypothesis and is therefore valid for unsteady compressible flows.

In an inertial reference frame, the conservation of momentum and mass gives (see nomenclature for the definitions):

$$
\int_{V} \frac{\partial \rho\left(\boldsymbol{q}-\boldsymbol{q}_{\infty}\right)}{\partial t} \mathrm{~d} V=-\int_{\partial V} \rho\left(\boldsymbol{q}-\boldsymbol{q}_{\infty}\right)(\boldsymbol{q} \cdot \boldsymbol{n}) \mathrm{d} S-\int_{\partial V}\left(p-p_{\infty}\right) \boldsymbol{n} \mathrm{d} S+\int_{\partial V}(\boldsymbol{\tau} \cdot \boldsymbol{n}) \mathrm{d} S
$$

This equation gives the balance between the aerodynamic force and the variation of fluid momentum. The drag is obtained by taking the $x$ component. Splitting the frontier of the domain $\partial V$ into the body surface $S_{a}$ and the outer surface $S_{e}$ (see figure 1):

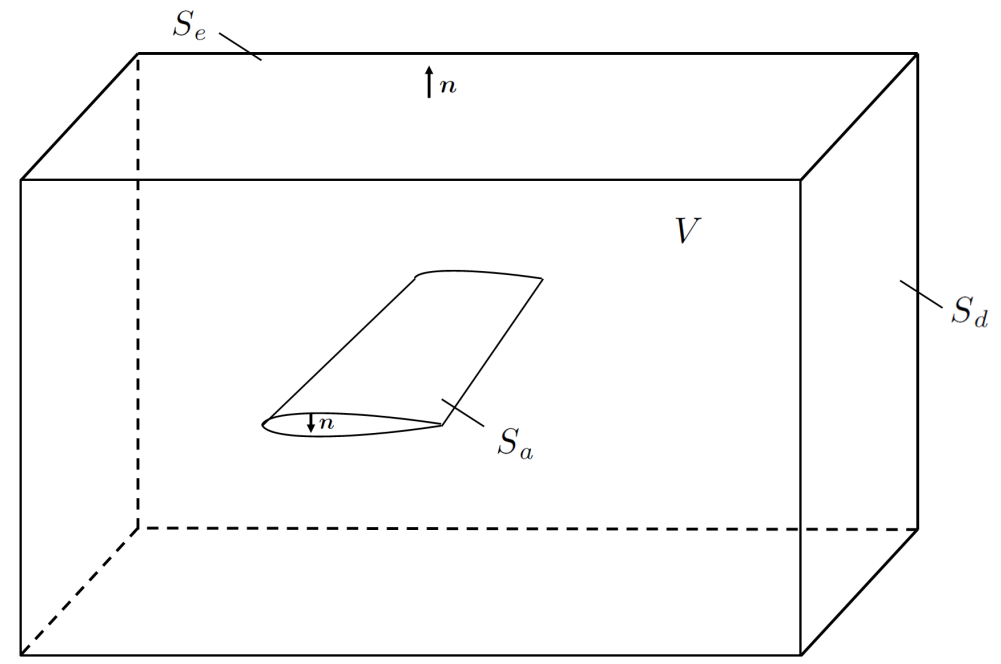

Figure 1. Control volume, surfaces and normal vectors

$$
\begin{aligned}
\int_{S_{a}}\left(\left(p-p_{\infty}\right)(\boldsymbol{i} \cdot \boldsymbol{n})-\left(\boldsymbol{\tau}_{\boldsymbol{x}} \cdot \boldsymbol{n}\right)\right) \mathrm{d} S & =\int_{S_{e}}\left(-\rho\left(u-u_{\infty}\right)(\boldsymbol{q} \cdot \boldsymbol{n})-\left(p-p_{\infty}\right)(\boldsymbol{i} \cdot \boldsymbol{n})+\left(\boldsymbol{\tau}_{\boldsymbol{x}} \cdot \boldsymbol{n}\right)\right) \mathrm{d} S \\
& -\int_{S_{a}} \rho\left(u-u_{\infty}\right)(\boldsymbol{q} \cdot \boldsymbol{n}) \mathrm{d} S-\int_{V} \frac{\partial \rho\left(u-u_{\infty}\right)}{\partial t} \mathrm{~d} V
\end{aligned}
$$

The left-hand side of the equation is the classic near-field drag, which can be broken down into pressure drag and friction drag.

$$
\begin{gathered}
D_{n f}=D_{p}+D_{f} \\
D_{p}=\int_{S_{a}}\left(p-p_{\infty}\right)(\boldsymbol{i} \cdot \boldsymbol{n}) \mathrm{d} S \\
D_{f}=\int_{S_{a}}-\left(\boldsymbol{\tau}_{\boldsymbol{x}} \cdot \boldsymbol{n}\right) \mathrm{d} S
\end{gathered}
$$


The right-hand side defines the far-field drag, which can be written:

$$
D_{f f}=\int_{S_{e}}(\boldsymbol{f} \cdot \boldsymbol{n}) \mathrm{d} S-\int_{S_{a}} \rho\left(u-u_{\infty}\right)(\boldsymbol{q} \cdot \boldsymbol{n}) \mathrm{d} S-\int_{V} \frac{\partial \rho\left(u-u_{\infty}\right)}{\partial t} \mathrm{~d} V
$$

with $\boldsymbol{f}=-\rho\left(u-u_{\infty}\right) \boldsymbol{q}-\left(p-p_{\infty}\right) \boldsymbol{i}+\boldsymbol{\tau}_{\boldsymbol{x}}$

The first surface term represents the flux of momentum through the outer surface and the forces applied on $S_{e}$. The second surface term is due to the motion of the body. The volume term accounts for the time dependence as well as the propagation in time of the momentum.

\section{B. Van der Vooren formulation (steady flows)}

For steady flows, the expression of far-field drag reduces to

$$
D_{f f}=\int_{S_{e}}(\boldsymbol{f} \cdot \boldsymbol{n}) \mathrm{d} S
$$

Upstream of the body, we have $u=u_{\infty}, p=p_{\infty}$ and $\boldsymbol{\tau}_{\boldsymbol{x}}=\mathbf{0}$. On the lateral surfaces sufficiently far or aligned with the streamlines, $\boldsymbol{q} \cdot \boldsymbol{n}=0, \boldsymbol{i} \cdot \boldsymbol{n}=0$ and $\boldsymbol{\tau}_{\boldsymbol{x}} \cdot \boldsymbol{n}=0$, so that

$$
D_{f f}=\int_{S_{d}}(\boldsymbol{f} \cdot \boldsymbol{n}) \mathrm{d} S
$$

Van der Vooren and Destarac formulation ${ }^{7}$ consists in breaking down the expression of $\boldsymbol{f}$ into an irreversible part and a reversible part. The notion of reversibility is not strictly rigorous since both stagnation enthalpy and entropy are involved, but the decomposition will be designated as such in this paper for the sake of simplicity.

On a wake plane $S_{d}$ sufficiently far from the drag sources, with a flow submitted to only irreversible processes, it is assumed that:

- $p=p_{\infty}$

- the velocity is parallel to $\boldsymbol{q}_{\infty}(v=w=0)$

Introducing these assumptions into $f$ and denoting $u_{i r r}$ the axial velocity on $S_{d}$, we get the irreversible part of $f$ :

$$
\boldsymbol{f}_{\text {irr }}=-\rho\left(u_{i r r}-u_{\infty}\right) \boldsymbol{q}+\boldsymbol{\tau}_{\boldsymbol{x}}
$$

The complementary is the reversible part:

$$
\boldsymbol{f}_{\text {rev }}=\boldsymbol{f}-\boldsymbol{f}_{\text {irr }}=-\rho\left(u-u_{\text {irr }}\right) \boldsymbol{q}-\left(p-p_{\infty}\right) \boldsymbol{i}
$$

The profile and induced drags can then be defined as:

$$
\begin{aligned}
D_{v w} & =\int_{S_{d}}\left(\boldsymbol{f}_{i r \boldsymbol{r}} \cdot \boldsymbol{n}\right) \mathrm{d} S \\
D_{i} & =\int_{S_{d}}\left(\boldsymbol{f}_{\text {rev }} \cdot \boldsymbol{n}\right) \mathrm{d} S
\end{aligned}
$$

The computation of $u_{i r r}$ starts from a general expression of the axial velocity as a function of the thermodynamic variables. Owing to the definitions of the stagnation enthalpy and the entropy defects:

$$
\begin{gathered}
\Delta H=H-H_{\infty} \\
\Delta s=\mathrm{C}_{\mathrm{p}} \ln \left(\frac{T_{\infty}}{T}\left(\frac{p}{p_{\infty}}\right)^{\frac{\gamma-1}{\gamma}}\right)
\end{gathered}
$$

the general expression of the axial velocity is therefore (without any hypothesis):

$$
u=u_{\infty} \sqrt{1+\frac{2 \Delta H}{u_{\infty}^{2}}-\frac{2}{(\gamma-1) M_{\infty}^{2}}\left(\mathrm{e}^{\frac{\gamma-1}{\gamma} \frac{\Delta s}{r}}\left(\frac{p}{p_{\infty}}\right)^{\frac{\gamma-1}{\gamma}}-1\right)-\frac{v^{2}+w^{2}}{u_{\infty}^{2}}}
$$


$u_{\text {irr }}$ (irreversible) can now be computed using the former assumptions:

$$
u_{i r r}=u_{\infty} \sqrt{1+\frac{2 \Delta H}{u_{\infty}^{2}}-\frac{2}{(\gamma-1) M_{\infty}^{2}}\left(\mathrm{e}^{\frac{\gamma-1}{\gamma} \frac{\Delta s}{r}}-1\right)}
$$

As pointed out by Méheut, ${ }^{9}$ this formulation is undefined if $p_{i} \leq p_{\infty}$. This situation can happen in the regions where the fluid is detached, where the vortices are strong, in the boundary layers if $M>M_{\infty}$ and downstream of internal shocks, which means inside almost any kind of drag source. For steady cases, the integration surface can be chosen around those undefined regions, resulting in small discrepancies on the drag coefficients. It is not as easy for unsteady flows. Gariépy therefore chose to use another definition of the axial velocity defect which is introduced in section II.D.1.

The induced drag is now fully defined:

$$
D_{i}=\int_{S_{d}}\left(\boldsymbol{f}_{\boldsymbol{r e v}} \cdot \boldsymbol{n}\right) \mathrm{d} S
$$

The profile drag has now to be further broken down into a wave and a viscous drag. $S_{d}$ can be switched again to $S_{e}$ :

$$
D_{v w}=\int_{S_{e}}\left(\boldsymbol{f}_{\text {irr }} \cdot \boldsymbol{n}\right) \mathrm{d} S
$$

The control volume can be split into a streamtube enclosing the shock $\Omega_{w}$, a streamtube enclosing the body and its boundary layer $\Omega_{v}$ and the rest of the volume $\Omega_{s p}$ (see figure 2). There is neither entropy nor stagnation enthalpy variations in $\Omega_{s p}$ as well as upstream of the sources so that the integral can be limited to the wake surfaces of the streamtubes using the divergence theorem:

$$
\begin{aligned}
& D_{v}=\int_{S_{v d}}\left(\boldsymbol{f}_{i r \boldsymbol{r}} \cdot \boldsymbol{n}\right) \mathrm{d} S \\
& D_{w}=\int_{S_{w d}}\left(\boldsymbol{f}_{\boldsymbol{i r r}} \cdot \boldsymbol{n}\right) \mathrm{d} S
\end{aligned}
$$

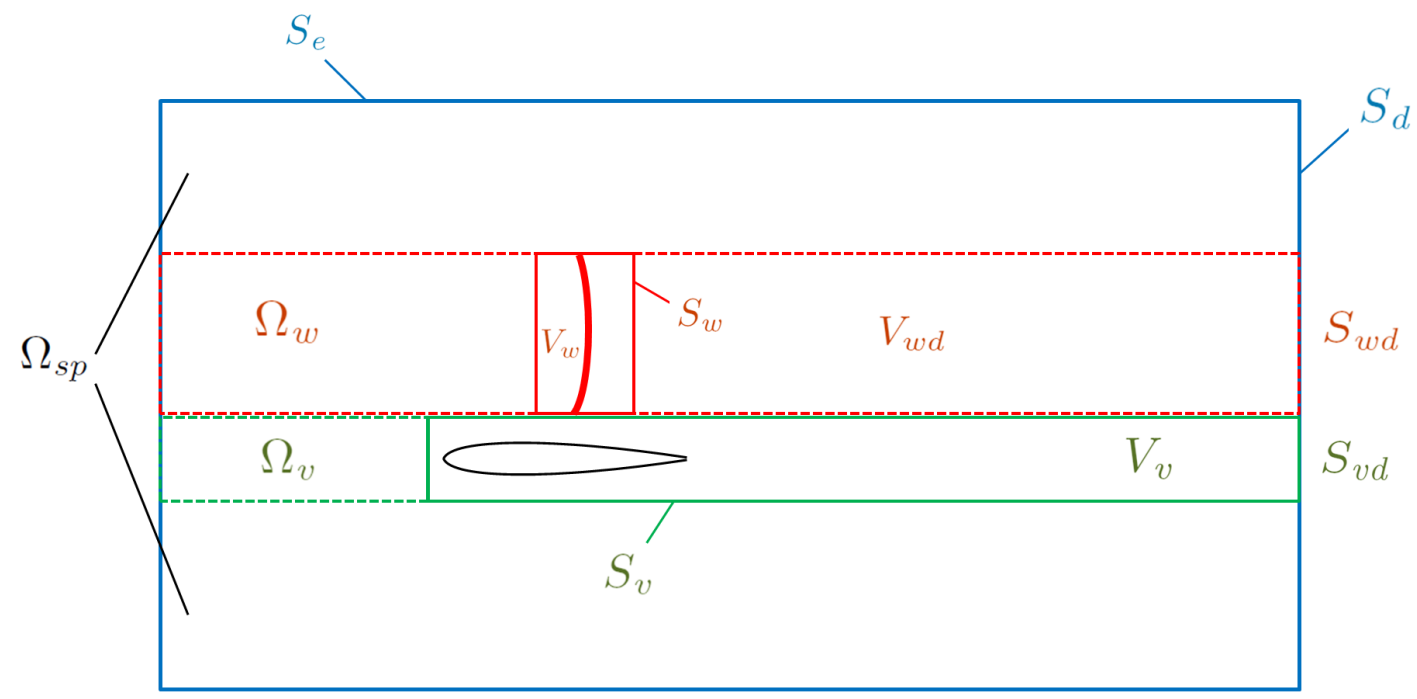

Figure 2. Integration volumes and surfaces

The viscous stresses can be neglected in $V_{w}+V_{w d}$. The wake surface of integration can now be moved closer to the source of wave drag for robustness and numerical accuracy. Denoting it $S_{w}$ and applying the divergence theorem in $V_{w d}$ :

$$
D_{w}=\int_{S_{w}}-\rho\left(u_{i r r}-u_{\infty}\right)(\boldsymbol{q} \cdot \boldsymbol{n}) \mathrm{d} S-\int_{V_{w d}} \nabla \cdot\left(\rho\left(u_{i r r}-u_{\infty}\right) \boldsymbol{q}\right) \mathrm{d} V
$$


Using the continuity equation $\boldsymbol{\nabla} \cdot(\rho \boldsymbol{q})=0$ :

$$
\boldsymbol{\nabla} \cdot\left(\rho\left(u_{i r r}-u_{\infty}\right) \boldsymbol{q}\right)=\rho \boldsymbol{q} \cdot \boldsymbol{\nabla}\left(u_{i r r}-u_{\infty}\right)=\rho \frac{\partial u_{i r r}}{\partial \Delta s} \boldsymbol{q} \cdot \boldsymbol{\nabla}(\Delta s)+\rho \frac{\partial u_{i r r}}{\partial \Delta H} \boldsymbol{q} \cdot \boldsymbol{\nabla}(\Delta H)
$$

It is assumed that the flow is isentropic and steady downstream of the shock so that both divergence terms are zero. The wave drag is finally:

$$
D_{w}=\int_{S_{w}}-\rho\left(u_{i r r}-u_{\infty}\right)(\boldsymbol{q} \cdot \boldsymbol{n}) \mathrm{d} S
$$

It is as if the entropy and stagnation enthalpy were probed on this surface close to the shock, but one must keep in mind that it really is an integration on a wake plane far from the sources of drag.

For convenience and since the integration is more reliable over a closed surface than on a wake plane, the wake integrals are turned into closed surface integrals as defined in figure 2. The last step is to compute the spurious drag as the difference between the near-field and the far-field drag. It contains a part of the numerical additive drag due to the numerical dissipation. The resulting formulation is the one exposed in Van der Vooren and Destarac paper ${ }^{7}$ (Note that a one vector formulation can be defined using the fact that $\boldsymbol{\nabla} \cdot \boldsymbol{f}=0$ everywhere.)

$$
\begin{gathered}
D_{w}=\int_{S_{w}}-\rho\left(u_{i r r}-u_{\infty}\right)(\boldsymbol{q} \cdot \boldsymbol{n}) \mathrm{d} S \\
D_{v}=\int_{S_{v}}\left(-\rho\left(u_{i r r}-u_{\infty}\right) \boldsymbol{q}+\boldsymbol{\tau}_{\boldsymbol{x}}\right) \cdot \boldsymbol{n} \mathrm{d} S \\
D_{i}=\int_{S_{e}}\left(-\rho\left(u-u_{i r r}\right) \boldsymbol{q}-\left(p-p_{\infty}\right) \boldsymbol{i}\right) \cdot \boldsymbol{n} \mathrm{d} S \\
D_{f f}=D_{w}+D_{v}+D_{i} \\
D_{s p}=D_{n f}-D_{f f}
\end{gathered}
$$

The derivation presented here differs from the ones currently used to demonstrate this decomposition in refs $^{6,7}$ for example. It however allows to introduce the unsteady problem more easily.

\section{Drag decomposition for unsteady flows}

The extension to unsteady flows of Van der Vooren theory is not as straightforward as it could seem. The approach presented here consists in first tackling the drag of each profile drag source isolated, before generalizing to a complete case.

\section{Unsteady wave drag expression}

Let us first consider the wave drag of an isolated normal shock moving in a perfect fluid. It is assumed that there is no reversible processes within the flow. We will again work in the streamtube $\Omega_{w}$ as defined for the steady case (see figure 2). The downstream irreversibilities will also be neglected here. The time-derivative term adds to the steady terms, so that the total drag, which is equal to the wave drag, is now:

$$
D=D_{w}=\int_{S_{w d}}-\rho\left(u-u_{\infty}\right)(\boldsymbol{q} \cdot \boldsymbol{n}) \mathrm{d} S-\int_{S_{w d}}\left(p-p_{\infty}\right)(\boldsymbol{i} \cdot \boldsymbol{n}) \mathrm{d} S-\int_{\Omega_{w}} \frac{\partial \rho\left(u-u_{\infty}\right)}{\partial t} \mathrm{~d} V
$$

The objective is now, similarly to the steady case, to move the integration surface $S_{w d}$ closer to the shock using the fact that the flow is assumed isentropic downstream of the shock. We choose $S_{w d}$ such that $p=p_{\infty}$ and $v=w=0$. We get the same irreversible velocity as in Eq. (16). The drag can now be expressed

$$
D_{w}=\int_{S_{w d}}-\rho\left(u_{i r r}-u_{\infty}\right)(\boldsymbol{q} \cdot \boldsymbol{n}) \mathrm{d} S-\int_{V_{w} \cup V_{w d}} \frac{\partial \rho\left(u-u_{\infty}\right)}{\partial t} \mathrm{~d} V
$$


gives:

If $V_{w}$ is the volume enclosed within $S_{w}$ (see figure 2), the application of the divergence theorem in $V_{w d}$

$$
\begin{aligned}
D_{w}= & \int_{S_{w}}-\rho\left(u_{i r r}-u_{\infty}\right)(\boldsymbol{q} \cdot \boldsymbol{n}) \mathrm{d} S-\int_{V_{w}} \frac{\partial \rho\left(u-u_{\infty}\right)}{\partial t} \mathrm{~d} V \\
& -\int_{V_{w d}}\left(\frac{\partial \rho\left(u-u_{\infty}\right)}{\partial t}+\boldsymbol{\nabla} \cdot\left(\rho\left(u_{i r r}-u_{\infty}\right) \boldsymbol{q}\right)\right) \mathrm{d} V
\end{aligned}
$$

Let us focus on the last term $I$, which can be written:

$$
I=-\int_{V_{w d}} \frac{\partial \rho\left(u-u_{i r r}\right)}{\partial t} \mathrm{~d} V-\int_{V_{w d}}\left(\frac{\partial \rho\left(u_{i r r}-u_{\infty}\right)}{\partial t}+\nabla \cdot\left(\rho\left(u_{i r r}-u_{\infty}\right) \boldsymbol{q}\right)\right) \mathrm{d} V
$$

Using the continuity equation $\frac{\partial \rho}{\partial t}+\boldsymbol{\nabla} \cdot(\rho \boldsymbol{q})=0$, it can be easily shown that

$$
\frac{\partial \rho\left(u_{i r r}-u_{\infty}\right)}{\partial t}+\nabla \cdot\left(\rho\left(u_{i r r}-u_{\infty}\right) \boldsymbol{q}\right)=\rho \frac{\mathrm{d}\left(u_{i r r}-u_{\infty}\right)}{\mathrm{d} t}=\rho\left(\frac{\mathrm{d} \Delta s}{\mathrm{~d} t} \frac{\partial u_{i r r}}{\partial \Delta s}+\frac{\mathrm{d} \Delta H}{\mathrm{~d} t} \frac{\partial u_{i r r}}{\partial \Delta H}\right)
$$

The flow is isentropic in $V_{w d}$ so $\frac{\mathrm{d} \Delta s}{\mathrm{~d} t}=0$ in $V_{w d}$. The second term is non-zero, contrarily to the steady case: $\frac{\mathrm{d} \Delta H}{\mathrm{~d} t}=\frac{1}{\rho} \frac{\partial p}{\partial t}$ and $\frac{\partial u_{i r r}}{\partial \Delta H}=\frac{1}{u_{i r r}}$. Finally:

$$
I=-\int_{V_{w d}}\left(\frac{\partial \rho\left(u-u_{i r r}\right)}{\partial t}+\frac{1}{u_{i r r}} \frac{\partial p}{\partial t}\right) \mathrm{d} V
$$

And the final expression for the wave drag is:

$$
D_{w}=\int_{S_{w}}-\rho\left(u_{i r r}-u_{\infty}\right)(\boldsymbol{q} \cdot \boldsymbol{n}) \mathrm{d} S-\int_{V_{w}} \frac{\partial \rho\left(u-u_{\infty}\right)}{\partial t} \mathrm{~d} V-\int_{V_{w d}}\left(\frac{\partial \rho\left(u-u_{i r r}\right)}{\partial t}+\frac{1}{u_{i r r}} \frac{\partial p}{\partial t}\right) \mathrm{d} V
$$

As a conclusion, the time derivative term around the shock area and in the downstream streamtube has been added so as to take the delay and the propagation of the source of drag into account. The manipulation made to integrate closer to the shock allows to get a small contribution of the wake (around $5 \%$ of the wave drag for the test case III.B.) and to reduce the numerical errors.

\section{Unsteady viscous drag expression}

We now consider a profile isolated in a flow, without shock waves and reversible processes. We work again in the streamtube $\Omega_{v}$, which can reduce again to $V_{v}$. The time-derivative term also adds to the steady term:

$$
D_{v}=\int_{S_{v}}\left(-\rho\left(u_{i r r}-u_{\infty}\right) \boldsymbol{q}+\boldsymbol{\tau}_{\boldsymbol{x}}\right) \cdot \boldsymbol{n} \mathrm{d} S-\int_{V_{v}} \frac{\partial \rho\left(u-u_{\infty}\right)}{\partial t} \mathrm{~d} V
$$

One could also move the wake surface a little upstream similarly to the shock case, the same volumic term would appear, but we do not judge it necessary here since the wake integral would remain large.

\section{Unsteady induced drag expression}

As for the steady case, the induced drag is here defined as the complementary part of the drag:

$$
\begin{aligned}
D_{i}= & \int_{S_{e}}\left(-\rho\left(u-u_{i r r}\right) \boldsymbol{q}-\left(p-p_{\infty}\right) \boldsymbol{i}\right) \cdot \boldsymbol{n} \mathrm{d} S \\
& -\int_{S_{d} \backslash\left(S_{w d}+S_{v d}\right)}-\rho\left(u_{i r r}-u_{\infty}\right)(\boldsymbol{q} \cdot \boldsymbol{n}) \mathrm{d} S-\int_{V \backslash\left(V_{w}+V_{w d}+V_{v}\right)} \frac{\partial \rho\left(u-u_{\infty}\right)}{\partial t} \mathrm{~d} V
\end{aligned}
$$

We call $V_{d}=V \backslash\left(V_{w} \cup V_{w d} \cup V_{v}\right)$. The second term can be transformed as usual by moving the surface $S_{d} \backslash\left(S_{w d} \cup S_{v d}\right)$ towards the upstream infinity. There $u_{i r r}=u_{\infty}$ so that only the volume integral remains:

$$
D_{i}=\int_{S_{e}}\left(-\rho\left(u-u_{i r r}\right) \boldsymbol{q}-\left(p-p_{\infty}\right) \boldsymbol{i}\right) \cdot \boldsymbol{n} \mathrm{d} S-\int_{V_{d}}\left(\frac{\partial \rho\left(u-u_{i r r}\right)}{\partial t}+\frac{1}{u_{i r r}} \frac{\partial p}{\partial t}\right) \mathrm{d} V
$$


If the fluid is inviscid without any shock, then $V_{d}$ becomes the whole volume and therefore the whole drag is taken into account. This expression of induced drag is not quite satisfactory, since it takes into account within the volume term phenomena which can be part of the irreversible drag. We will focus in the applications on analyzing its behavior.

\section{Final decomposition}

One last term is to be added to this decomposition: $-\int_{S_{a}} \rho\left(u-u_{\infty}\right)(\boldsymbol{q} \cdot \boldsymbol{n}) \mathrm{d} S$ from Eq. (6) is simply the drag/thrust due to the motion of the body in an inertial reference frame. It is equal to zero if the body is motionless and the unsteadiness of the flow is natural.

The final decomposition is therefore:

$$
\begin{gathered}
D_{w}=\int_{S_{w}}-\rho\left(u_{i r r}-u_{\infty}\right)(\boldsymbol{q} \cdot \boldsymbol{n}) \mathrm{d} S-\int_{V_{w}} \frac{\partial \rho\left(u-u_{\infty}\right)}{\partial t} \mathrm{~d} V-\int_{V_{w d}}\left(\frac{\partial \rho\left(u-u_{i r r}\right)}{\partial t}+\frac{1}{u_{i r r}} \frac{\partial p}{\partial t}\right) \mathrm{d} V \\
D_{v}=\int_{S_{v}}\left(-\rho\left(u_{i r r}-u_{\infty}\right) \boldsymbol{q}+\boldsymbol{\tau}_{\boldsymbol{x}}\right) \cdot \boldsymbol{n} \mathrm{d} S-\int_{V_{v}} \frac{\partial \rho\left(u-u_{\infty}\right)}{\partial t} \mathrm{~d} V \\
D_{i}=\int_{S_{e}}\left(-\rho\left(u-u_{i r r}\right) \boldsymbol{q}-\left(p-p_{\infty}\right) \boldsymbol{i}\right) \cdot \boldsymbol{n} \mathrm{d} S-\int_{V_{d}}\left(\frac{\partial \rho\left(u-u_{i r r}\right)}{\partial t}+\frac{1}{u_{i r r}} \frac{\partial p}{\partial t}\right) \mathrm{d} V \\
D_{m}=\int_{S_{a}}-\rho\left(u-u_{\infty}\right)(\boldsymbol{q} \cdot \boldsymbol{n}) \mathrm{d} S \\
D_{f f}=D_{w}+D_{v}+D_{i}+D_{m} \\
D_{s p}=D_{n f}-D_{f f}
\end{gathered}
$$

Let us make some comments:

- If we remove all the time-derivative terms, we get the steady formulations as expressed in II.B.

- The zones where $u_{i r r}$ is not defined are usually concentrated in $V_{v}$ and $V_{w}$ so that we can still use Van der Vooren definition in most cases. However, we give in II.D.1 another decomposition of $u-u_{\infty}$ which is defined everywhere in the fluid and used by Gariépy in his paper, ${ }^{6}$ and might be required for certain configurations.

\section{Gariépy decomposition}

Let us now analyze and compare the formulation suggested by Gariépy. ${ }^{6}$ He uses another definition of the axial velocity defect first introduced in Ref. 9 which seems better suited to unsteady flows since it is defined everywhere.

\section{A new decomposition of the axial velocity defect}

Méheut ${ }^{9}$ introduced another decomposition of $u-u_{\infty}$. His demonstration consists in tackling the problem the other way around: he asserts that the flow is reversible, which means isentropic, in order to get a reversible axial velocity $u_{\text {rev }}$ as a function of $p, v$ and $w$ in the reversible case. Starting from equation (15) and assuming that on a wake plane $S_{d}$ :

- $\Delta H=0$

- $\Delta s=0$

We get the reversible velocity:

$$
u_{\text {rev }}=u_{\infty} \sqrt{1-\frac{2}{(\gamma-1) M_{\infty}^{2}}\left(\left(\frac{p}{p_{\infty}}\right)^{\frac{\gamma-1}{\gamma}}-1\right)-\frac{v^{2}+w^{2}}{u_{\infty}^{2}}}
$$

We now define $\boldsymbol{f}_{\text {rev }}$ as:

$$
\boldsymbol{f}_{\text {rev }}=-\rho\left(u_{\text {rev }}-u_{\infty}\right) \boldsymbol{q}-\left(p-p_{\infty}\right) \boldsymbol{i}
$$


The irreversible part is now the complementary part:

$$
\boldsymbol{f}_{\text {irr }}=-\rho\left(u-u_{\text {rev }}\right) \boldsymbol{q}+\boldsymbol{\tau}_{\boldsymbol{x}}
$$

The drag coefficients are still defined in the same way, but in order to move the surface of integration closer to the shock for $D_{w}$, it is assumed that $u-u_{\text {rev }}$ depends only on $\Delta s$ and $\Delta H$, which is only an approximation. It is therefore expected that the behavior of this formulation is less robust than the one from Van der Vooren.

Gariépy asserts in his $\operatorname{article}^{8}$ that $\Delta H$ should be taken into account in $u_{\text {rev }}$, which is equivalent to withdrawing it from $u_{i r r}$. It is of small consequences for steady test cases (a few drag points at most). It

may however become very large for unsteady cases, since the stagnation enthalpy varies strongly versus time. We will denote them $u_{r e v}^{*}$ and $u_{i r r}^{*}$ and add a ${ }^{*}$ superscript to the corresponding variables.

$$
\begin{gathered}
u_{r e v}^{*}=u_{\infty} \sqrt{1+\frac{2 \Delta H}{u_{\infty}^{2}}-\frac{2}{(\gamma-1) M_{\infty}^{2}}\left(\left(\frac{p}{p_{\infty}}\right)^{\frac{\gamma-1}{\gamma}}-1\right)-\frac{v^{2}+w^{2}}{u_{\infty}^{2}}} \\
u_{i r r}^{*}=u_{\infty} \sqrt{1-\frac{2}{(\gamma-1) M_{\infty}^{2}}\left(\mathrm{e}^{\frac{\gamma-1}{\gamma} \frac{\Delta s}{r}}-1\right)}
\end{gathered}
$$

There is no theoretical motivation to remove the stagnation enthalpy from $u_{i r r}$, this is why we kept this term in our evaluation of $u_{i r r}$. The variation of stagnation enthalpy due to the unsteadiness of a shock should for example appear inside the wave drag. All three formulations, the one proposed in II.B. and the present ones with $u_{r e v}$ and $u_{r e v}^{*}$, will be compared for steady applications in section III.A.

\section{Gariépy unsteady decomposition}

Gariépy ${ }^{6}$ introduces this new definition within the steady decomposition and suggests to include the time derivatives into an unsteady drag coefficient. His equations are written in the relative reference frame but were here translated into the inertial reference frame for comparison. His formulation can be written as follows:

$$
\begin{gathered}
D_{w}^{G}=\int_{S_{w}}-\rho\left(u-u_{r e v}^{*}\right)(\boldsymbol{q} \cdot \boldsymbol{n}) \mathrm{d} S \\
D_{v}^{G}=\int_{S_{v}}\left(-\rho\left(u-u_{r e v}^{*}\right) \boldsymbol{q}+\boldsymbol{\tau}_{\boldsymbol{x}}\right) \cdot \boldsymbol{n} \mathrm{d} S \\
D_{i}^{G}=\int_{S_{e}}\left(-\rho\left(u_{r e v}-u_{\infty}\right) \boldsymbol{q}-\left(p-p_{\infty}\right) \boldsymbol{i}\right) \cdot \boldsymbol{n} \mathrm{d} S \\
D_{u n s}^{G}=\int_{S_{e}}-\rho\left(u_{r e v}^{*}-u_{r e v}\right)(\boldsymbol{q} \cdot \boldsymbol{n}) \mathrm{d} S-\int_{V} \frac{\partial \rho\left(u-u_{\infty}\right)}{\partial t} \mathrm{~d} V \\
D_{s p}^{G}=\int_{V_{d} \cup V_{w d}} \boldsymbol{\nabla} \cdot\left(-\rho\left(u-u_{r e v}^{*}\right) \boldsymbol{q}+\boldsymbol{\tau}_{\boldsymbol{x}}\right) \mathrm{d} V \\
D_{m}=\int_{S_{a}}-\rho\left(u-u_{\infty}\right)(\boldsymbol{q} \cdot \boldsymbol{n}) \mathrm{d} S \\
D_{f f}^{G}=D_{w}^{G}+D_{v}^{G}+D_{i}^{G}+D_{u n s}^{G}+D_{s p}^{G}+D_{m}
\end{gathered}
$$

Here are some remarks about this splitting:

- The so-called unsteady drag $D_{u n s}^{G}$ is not directly related to physical sources of drag.

- The reversible velocity $u_{r e v}^{*}$ is used, which is equivalent to not taking the stagnation enthalpy variations into the profile drag coefficients. The motivation is that the variations of $H$ in time are due to the unsteadiness of the flow only. It is true but we consider that this time variation is bound to the movement of a shock for example and should be included in the unsteady wave drag coefficient. 
- According to Ref. $6, \boldsymbol{\nabla} \cdot \boldsymbol{f}_{i r r}^{*}$ would be located only in the regions of production of irreversible drag (shocks, boundary layers and wakes), but it is not always the case. The wave and viscous drag coefficients are in fact strongly dependent on the integration volume chosen and the spurious drag can be large with this definition. Note also that the balance between far-field and near-field drag is not ensured.

Both formulations have been implemented and are compared in section III.

\section{Applications}

The formulations using $u_{i r r}, u_{\text {rev }}$ and $u_{r e v}^{*}$ are first applied to steady test cases. They are then tested on three unsteady flow test cases which allow drawing some conclusions about the validity of the approach. The Onera code elsA ${ }^{10}$ is used for every flow computation. Jameson numerical scheme and the turbulence model of Spalart-Allmaras are used except for the second unsteady case. The unsteady computations are URANS calculations.

\section{A. Comparative study of the expressions using $u_{i r r}, u_{r e v}$ and $u_{r e v}^{*}$ on steady flows}

The different expressions available for the computation of the axial velocity defect have been compared in many steady cases, Euler, RANS, 2D and 3D, with or without angle of attack. The effect of the downstream extension of the integration surfaces is studied. The aim is to check the validity of the use of $u_{r e v}$ and $u_{r e v}^{*}$ compared to $u_{i r r}$ as we change the integration domain. All cases gave the same conclusions, which we can summarize with a general case of a 3D wing in a transonic flow with a non zero angle of attack.

The wing is a rectangular NACA0012-based wing. The mesh is around 1 million nodes and is shown in figure 3(a). The aerodynamic conditions are: $M_{\infty}=0.8, \alpha=2.5 \mathrm{deg}$ and $R e=3 \times 10^{6}$. The convergence curves in figure $3(\mathrm{~b})$ show that the computation is very well converged after the 10,000 iterations. The variation of the near-field drag coefficient (in blue) in particular is less than a thousandth of drag count.

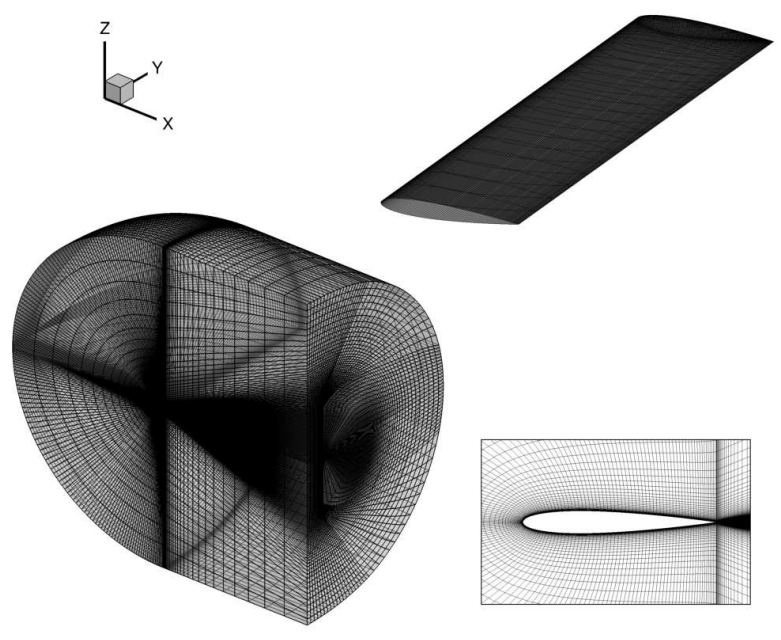

(a) Visualization of the mesh

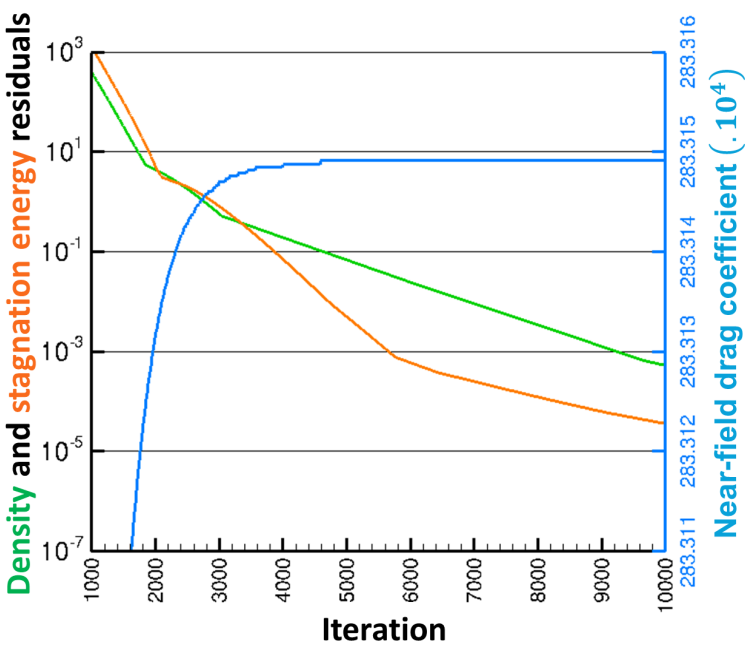

(b) Convergence curves

Figure 3. Mesh and convergence curves of steady 3D NACA0012 wing at $M_{\infty}=0.8, \alpha=2.5 \mathrm{deg}, R e=3 \times 10^{6}$.

The flow field is presented in figure 4(a). The shock wave on the upper side is quite strong and a vortex appears at the tip of the wing. The flow field is post-processed after 10,000 iterations by the in-house code ffd72. The first step of far-field drag extraction is the definition of the integration surfaces using volume criteria. Those criteria are described in Van der Vooren and Destarac paper. ${ }^{7}$ The downstream extension of the integration surfaces can vary at the demand of the user. Figure 4(b) shows an example of the integration surfaces for a given downstream extension. 


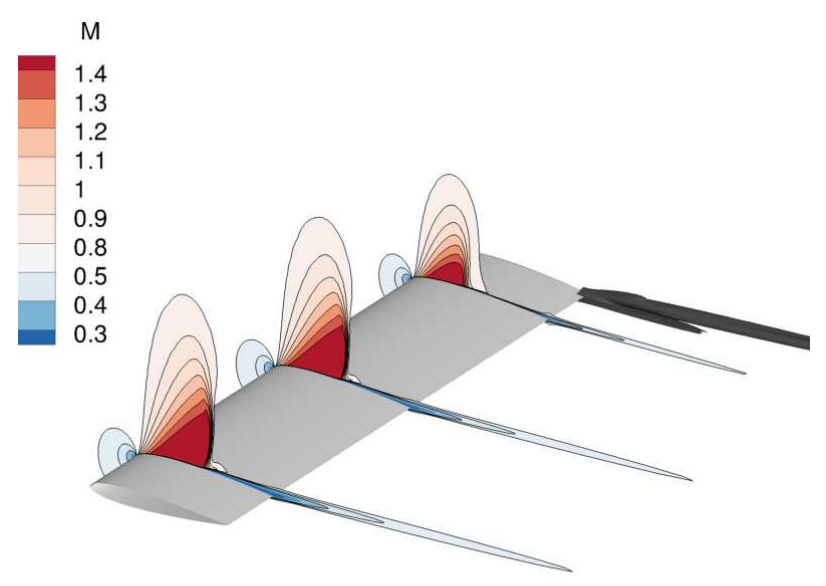

(a) Mach contours and iso-surface of vorticity

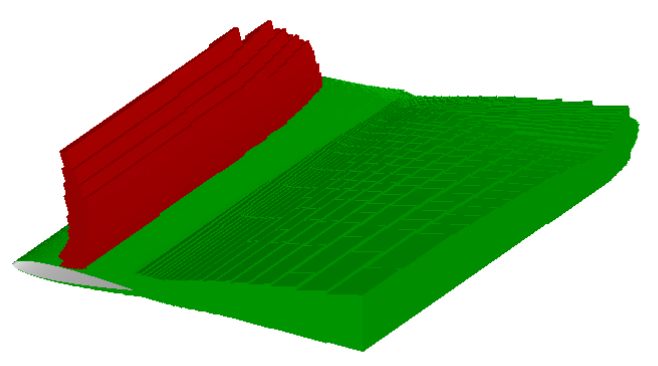

(b) Integration surfaces (red=shock, green=viscous)

Figure 4. Flow field and integration surfaces of steady 3D NACA0012 wing at $M_{\infty}=0.8, \alpha=2.5 \mathrm{deg}, R e=3 \times 10^{6}$.

Once the surfaces defined, the drag coefficients are computed by ffd 72 . The downstream extension of the surfaces was set to vary in figure 5, allowing a comparison between the three expressions of the axial velocity defect. The classical expression using $u_{i r r}$ as defined by Van der Vooren and Destarac is quite reliable, even very close to the sources of drag. The expressions with $u_{r e v}$ and $u_{r e v}^{*}$ give less satisfactory results. These observations are consistent with the theoretical remarks made in sections II.A. and II.D.1. Another comment is that there is very little difference between $u_{r e v}$ and $u_{r e v}^{*}$ in the steady case. It will not be the case for unsteady applications.

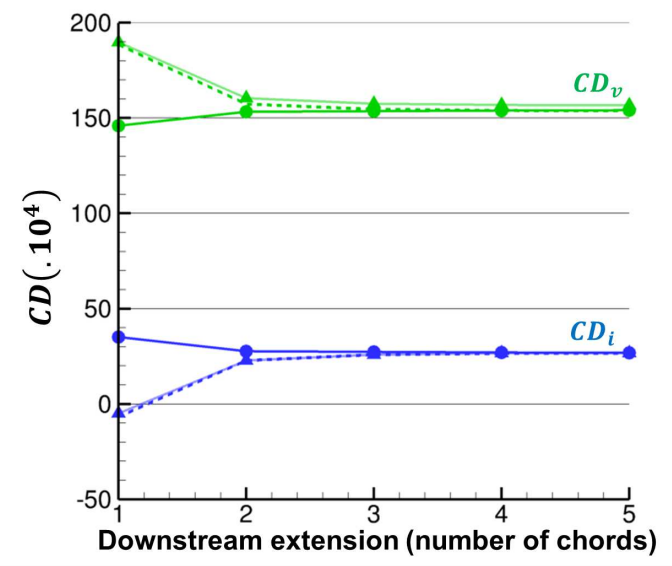

(a) Viscous and induced drag coefficients

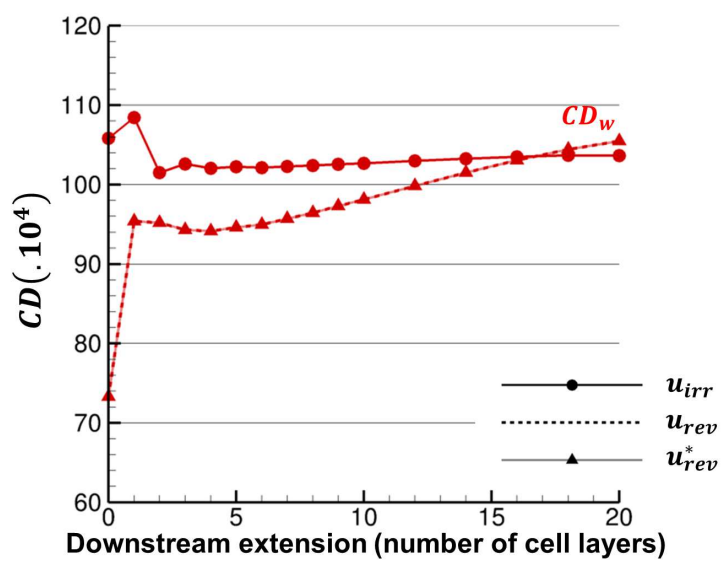

(b) Wave drag coefficient

Figure 5. Evolution of the wave, viscous and induced drag with respect to the downstream extension of the integration surfaces.

The conclusion of this first study is that the integration volumes and surfaces must be chosen very carefully. We will also try to use as much as possible $u_{i r r}$ instead of $u_{\text {rev }}$ in the following applications. 


\section{B. Buffeting}

The first unsteady test case is an OAT15A profile under buffeting conditions. The mesh is 2D with around 300,000 nodes (see figure 6(a)). The study was carried out over one period, with a time step of $1.7 \times 10^{-5}$, which corresponds to 1,000 steps by period. The Mach number is $M_{\infty}=0.73, \alpha=4.5 \mathrm{deg}$ and $R e=13 \times 10^{6}$. The unsteady computation was converged over several periods in order to reach the full periodicity and avoid the transient phenomena. The curve of lift vs drag over one period is then almost closed as shown in figure 6(b).

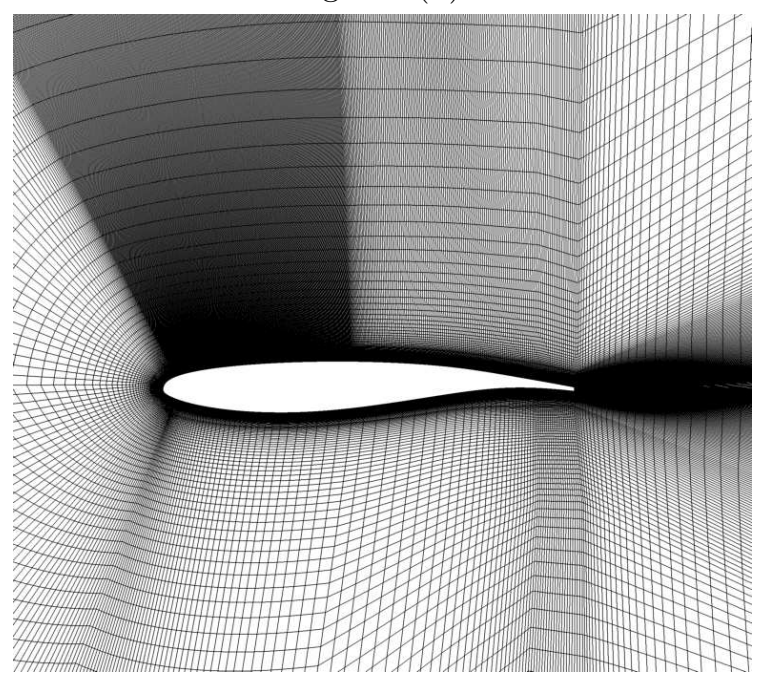

(a) Visualization of the mesh

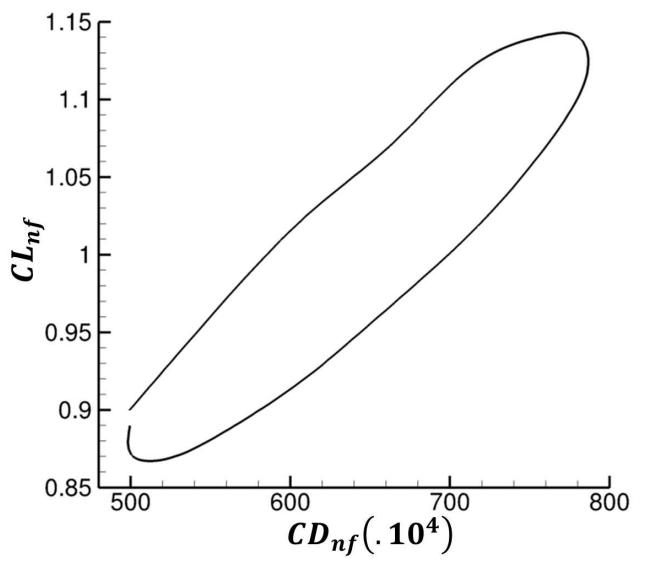

(b) Near field drag vs near field lift over one period

Figure 6. Mesh and lift/drag polar of the natural buffeting over an OAT15A profile at $M_{\infty}=0.73, \alpha=4.5$ deg, $R e=13 \times 10^{6}$.

Figure 7(a) shows the instantaneous flow field taken when the shock is in the downstream position at time step 500. The integration surfaces computed by ffd72 at the same time step are shown in figure 7(b). Recall that the integration surface for the induced drag is the outer surface, and $V_{d}$ is the complementary of the volumes shown in figure $7(\mathrm{~b})$ in the whole domain.

The drag extraction was carried out over one period. The resulting time evolutions are shown in figure 8. The formulation developed and described in this paper is in solid lines. The total far-field drag (pink) increases while the shock moves downstream and decreases while the shock moves upstream. It is in good agreement with the near-field drag. The spurious drag (orange) is indeed at most 20 drag counts or $2 \%$ of the total drag. The validation of the other drag coefficients can only be qualitative by analyzing the flow field. The components will also be compared to those obtained using Gariépy's formulation.

The wave drag (red) takes values between 170 and 280 drag counts. It is strongest when the shock is at the most downstream position, as expected. The viscous drag (green) varies between 200 and 550 drag counts. It is strongest when the shock is close to the leading edge and the flow separation is the strongest. The time evolution seems therefore valid. What is noticeable is the strong induced drag (blue) which oscillates between negative and positive values. The induced drag is zero for steady $2 \mathrm{D}$ configurations. For unsteady cases however, vorticity is continuously shed in the wake, leading to variations in the lift and therefore in the induced drag, which might explain the evolution observed.

Gariépy's formulation results are presented in dashed lines, with the same colors. It was found that the choice of the integration surfaces, in particular the downstream extension of the viscous volume, had a very strong impact on the results. The downstream extension was chosen of 3 chords in order to get the least spurious drag. The far-field drag is equal to the former one since only the decomposition is different. The spurious drag however differs since it is not computed as a balance. It is actually rather strong, at most $12 \%$ of the total drag. The wave and viscous drag coefficients have the same order of magnitude but are in phase opposition with our new formulation. The induced drag is quite small and constant in time, which could be expected for a 2D profile. The unsteady drag coefficient (light blue) on the contrary is strong with positive and negative values. It is remarkable that this unsteady coefficient is rather close to our induced coefficient computed with our new formulation. The new induced drag is indeed mainly due to the unsteadiness. 


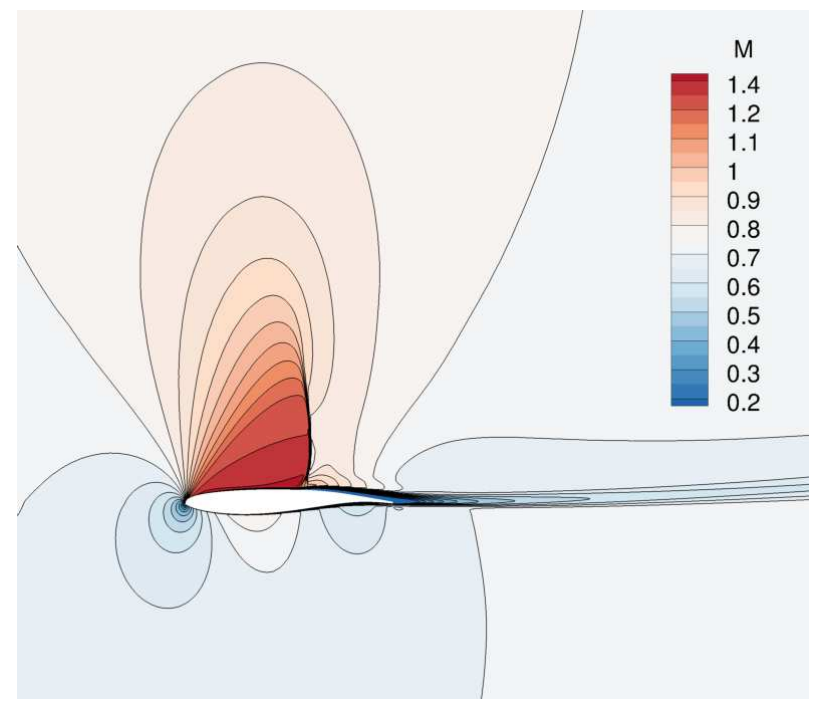

(a) Mach contours

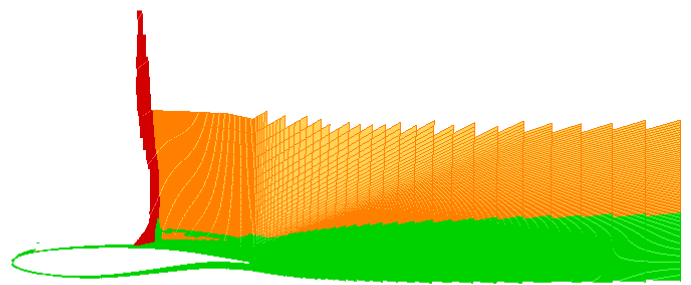

(b) Integration surfaces (red=shock, orange $=$ shock wake, green $=$ viscous

Figure 7. Flow field and integration surfaces taken at time step 500 of the natural buffeting on an OAT15A profile at $M_{\infty}=0.73, \alpha=4.5 \mathrm{deg}, \operatorname{Re}=13 \times 10^{6}$.

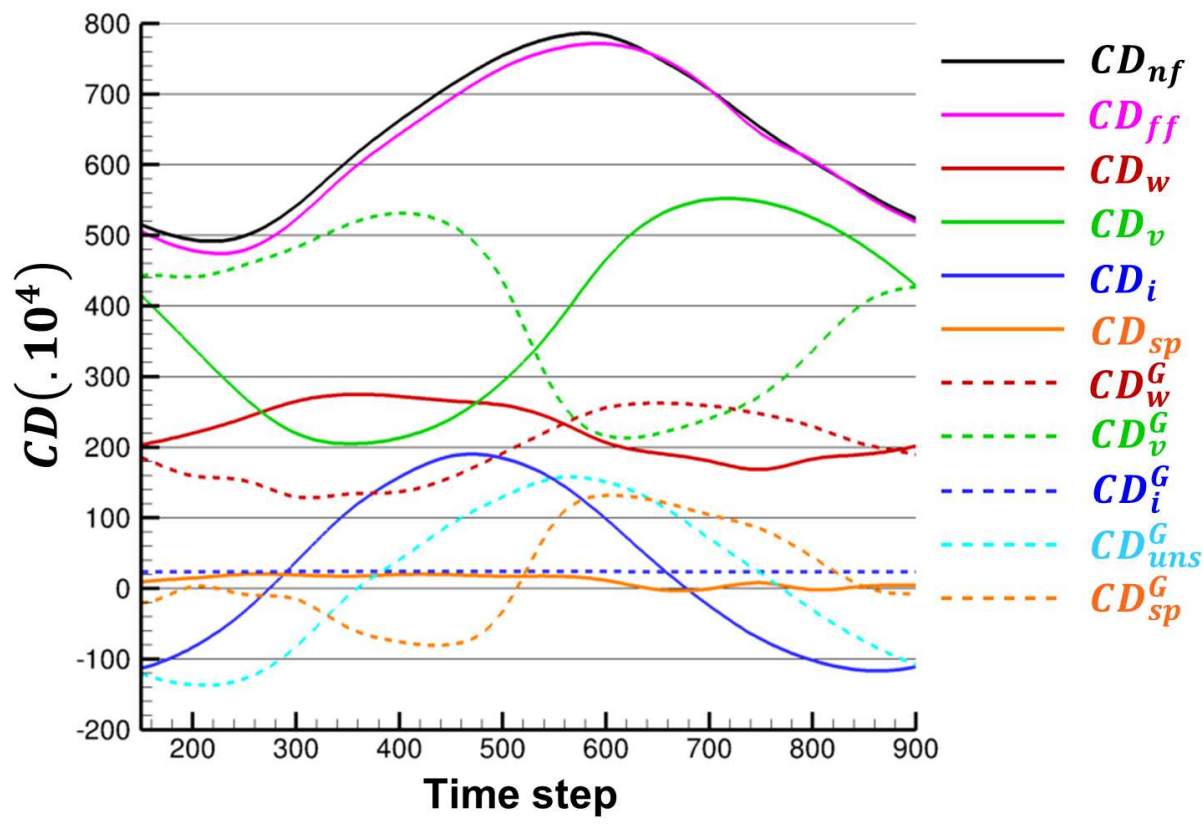

Figure 8. Evolution of the drag coefficients with respect to time over one period. 
The conclusion of this first test case is that our unsteady formulation gives good results despite the discrepancies with the only other unsteady decomposition available. The fact that the new formulation shows a correlation of the wave drag coefficient with the intensity of the shock, contrarily to Gariépy's formulation, is encouraging. Further validation regarding the induced drag expression is however required.

\section{Vortex shedding}

The second unsteady case is the natural vortex shedding downstream of a NACA0012 profile at $\alpha=20$ deg at low Mach number $M_{\infty}=0.2$ and $R e=2 \times 10^{6}$. The turbulence model is here $k-\omega$ and the numerical scheme is AUSM-P. The far-field drag reduces to viscous and induced drags, allowing a better understanding of the decomposition.

The mesh is a 550,000 nodes 2D mesh (see figure 9(a)). The time step is $5 \times 10^{-6}$, which corresponds to 3,000 steps by period. Here again several periods were simulated before extracting the flow field and the periodic state was ensured looking at lift vs drag curves as in figure 9(b).

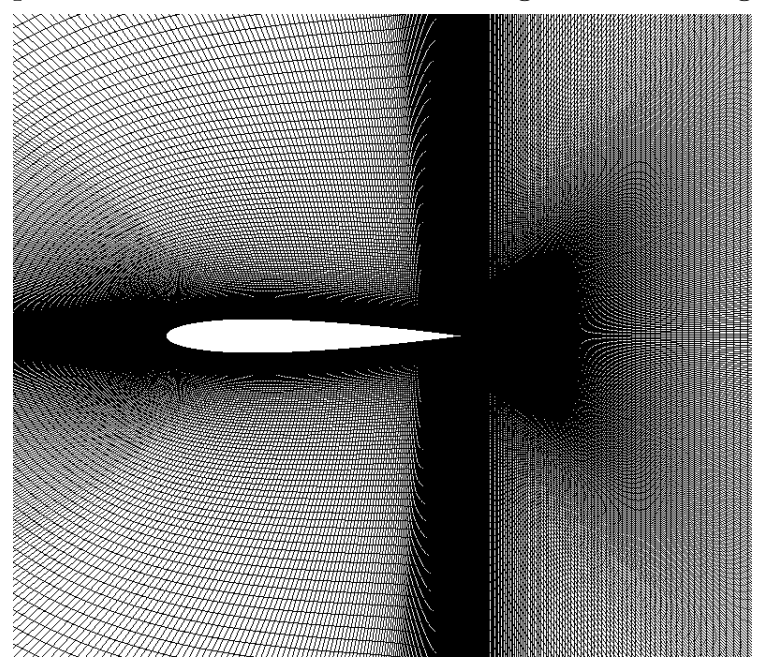

(a) Visualization of the mesh

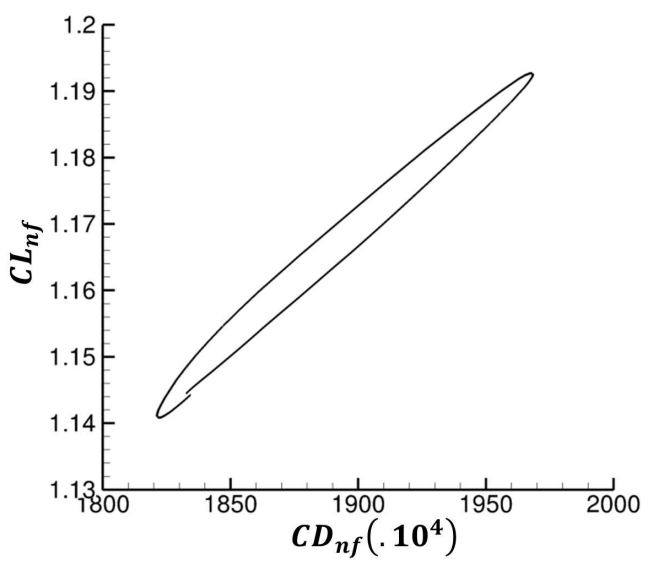

(b) Near field drag vs near field lift over one period

Figure 9. Mesh and lift/drag polar of the natural unsteady vortex shedding downstream of a NACA0012 profile at $M_{\infty}=0.2, \alpha=20 \mathrm{deg}, R e=2 \times 10^{6}$.

The flow field is presented in figure 10(a). Two vortices are emitted periodically and are then advected along the wake. The integration volumes are computed at each extraction of drag. An example of the viscous volume is shown in figure 10(b). The induced surface is there again the outer surface and the integration volume is the complementary of the viscous volume.

The drag extraction is carried out over one period. The resulting time evolution curves are shown in figure 11. The far-field drag (pink) is in good agreement with the near-field drag (black). Their peaks match the instant when a pair of vortices is released. The high level of drag is consistent with the experimental results of Mesquita. ${ }^{11}$

The results of the new formulation are presented in solid lines. The viscous drag (green) is the strongest when the separation occurs. The induced drag (blue) is here also non zero. However in this case it remains positive with relatively small values. Its variations are consistent with the variations of vorticity which is created at the separation instant. We could have expected it to vary in negative and positive values as in the previous test case. It is difficult to find a correct explanation since the wake is very wide, resulting in a non obvious split of the viscous and induced drags. The spurious drag (orange) is rather small, around 1\% of the total drag.

Gariépy's formulation is presented in dashed lines. The volumes were also chosen so as to get the least spurious drag. It is however stronger, at most 100 drag counts or $5 \%$ of the total drag. The unsteady drag term is also quite strong. The induced drag is almost zero and negative and the viscous drag is under estimated compared to our formulation.

This second test case therefore confirms the first one: the new formulation gives satisfactory drag coefficient evolutions even though the induced drag coefficient requires further investigations. 


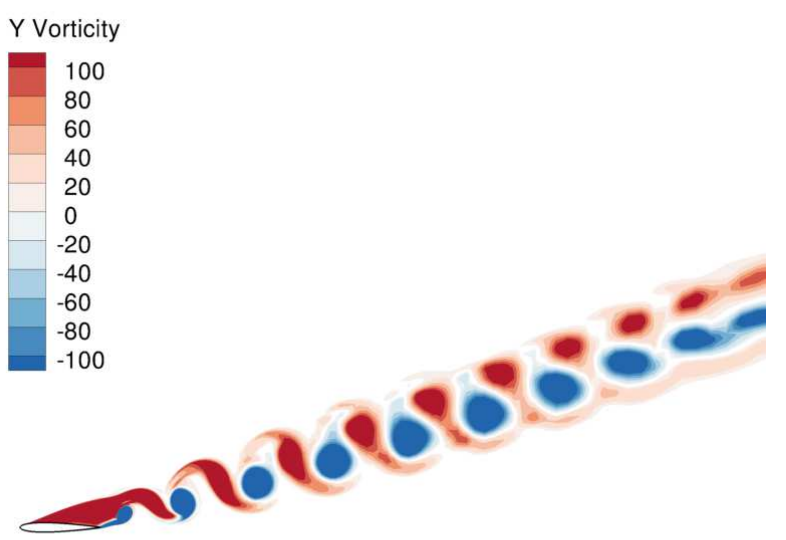

(a) Vorticity contours

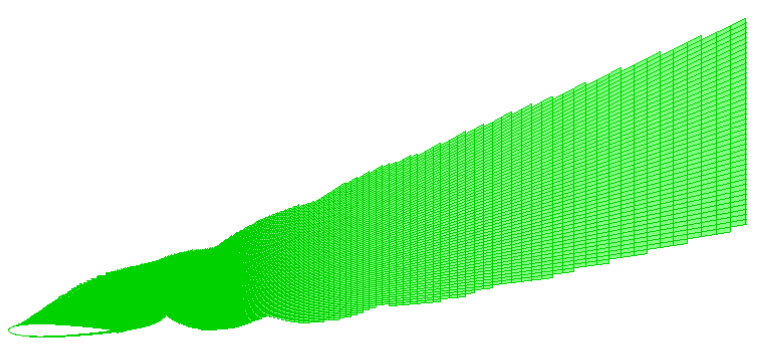

(b) Viscous integration surface

Figure 10. Flow field and integration surface taken at time step 2000 of the natural unsteady vortex shedding downstream of a NACA0012 profile at $M_{\infty}=0.2, \alpha=20 \mathrm{deg}, R e=2 \times 10^{6}$.

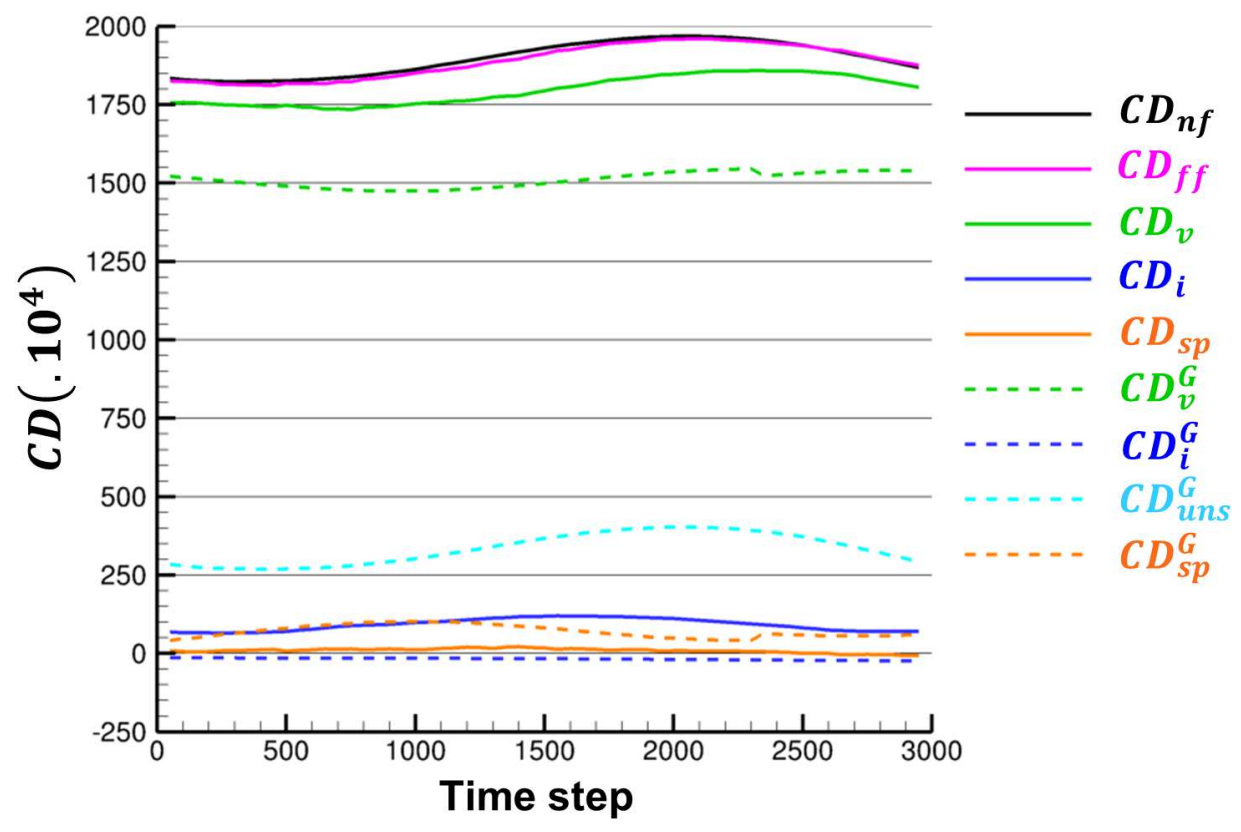

Figure 11. Evolution of the drag coefficients with respect to time over one period. 


\section{Oscillating profile}

In order to validate the decomposition between induced and viscous drag coefficients, a last test case is studied here: a NACA0012 oscillating profile. It is the same configuration as the last case of Gariépy's paper. ${ }^{6}$ The profile is oscillating between -5 and +5 deg at the reduced frequency of 0.1 . The Mach number is $M_{\infty}=0.3$ and the Reynolds number is $R e=6.6 \times 10^{6}$. The time step chosen is $1.16 \times 10^{-2}$, which corresponds to 9,000 steps by period.

The grid used here is a 140,000 nodes 2D mesh as shown in figure 12(a). The polar of lift vs drag after several oscillations is a closed loop, as seen in figure 12(b), so that the periodicity of the flow is considered reached.

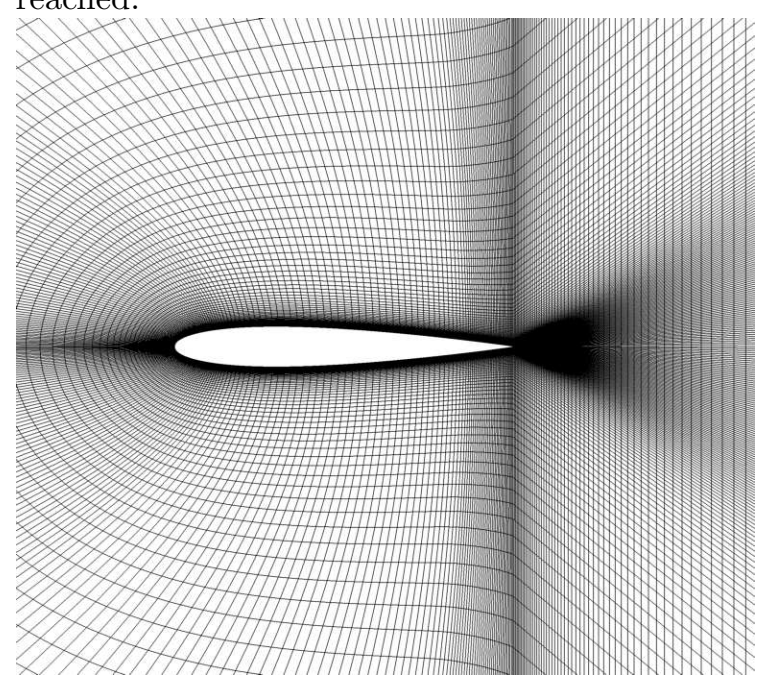

(a) Visualization of the mesh

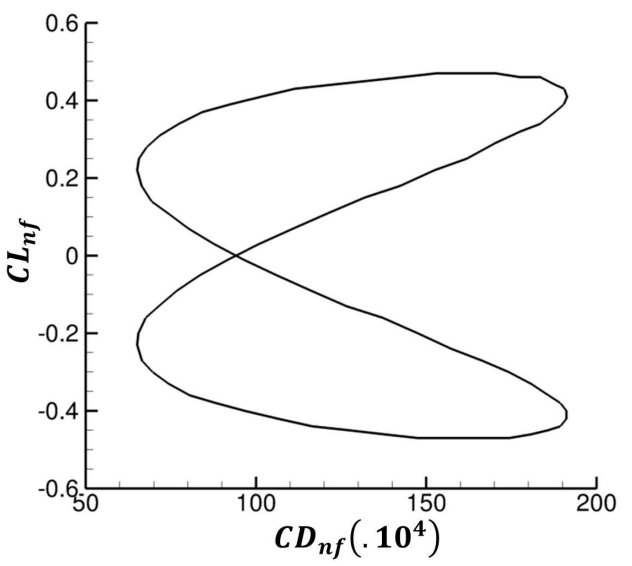

(b) Near field drag vs near field lift over one period

Figure 12. Mesh and lift/drag polar of the oscillating NACA0012 profile at $M_{\infty}=0.3, R e=6.6 \times 10^{6}$.

A snapshot of the flowfield is shown in figure 13(a). There is no flow separation even at the highest angles of attack. The integration volume at the same instant for the viscous drag is presented in figure 13(b). It matches the boundary layer and the wake nicely. The downstream extension for the far-field drag integration was chosen at 3 chords given the quality of the mesh in the wake.

The curves of evolution of the drag as a function of time are presented in figure 14. The total drag over one period has two peaks, one for each maximal angle $(-5 \mathrm{deg}$ and $+5 \mathrm{deg})$ and varies between 65 and 190 drag counts. The far-field drag (pink) is again in very good agreement with the near-field drag (black): both curves are almost superimposed. The results are also consistent with Gariépy's paper. ${ }^{6}$ We can however notice that the time evolutions are slightly in advance on the angle of attack. The lift drag in red is however very slightly delayed on the angle of attack, which may explain this time shift. The motion drag in grey (hardly visible behind the orange spurious drag) is almost zero, since the velocity on the upper side projected on the normal vector cancels out with the lower side.

The solid lines stand for the new formulation. The viscous drag in green varies around 100 drag counts. It also shows two peaks which are quite in phase with the angle of attack. The variations are not so strong since there is no separation of the flow and the angle of attack remains small. The induced drag (blue) on the other hand is again very strong, taking positive and negative values between -30 and 85 drag counts. The negative values can be understood as a thrust when the profile is moving upwards or downwards. The spurious drag is less than one drag count.

As for Gariépy's decomposition, difficulties were encountered with the choice of the integration volumes. The domain had to be reduced to five chords in all directions, and three in the downstream direction, in order to match the far-field with the near-field. The discrepancy is probably due to the relatively poor quality of the mesh. Computation on a finer mesh is ongoing. Our new formulation however proved to be quite robust to any choice of domain extent. Another difficulty occurs when trying to retrieve the results shown by Gariépy in his paper. The choice of the integration volumes has indeed a great influence on the results and they were not specified in the original paper. The results presented here are the best achieved in this study. The viscous drag in dashed green line is stronger, varying little around 100 drag counts instead 


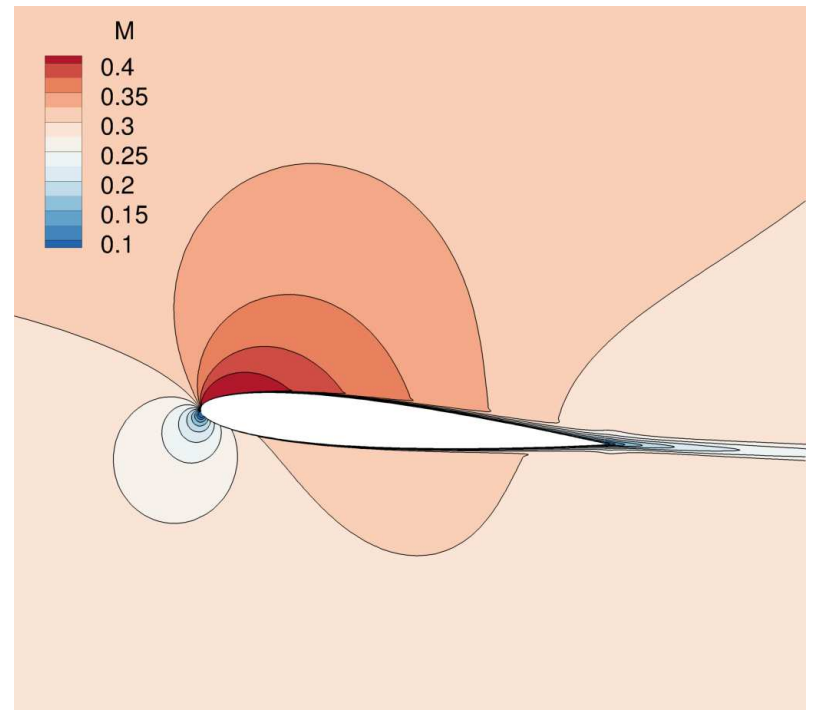

(a) Mach contours (b) Viscous integration surface

Figure 13. Flow field and integration surface taken at time step 2250 (5 deg) of the oscillating NACA0012 profile at $M_{\infty}=0.3, R e=6.6 \times 10^{6}$.
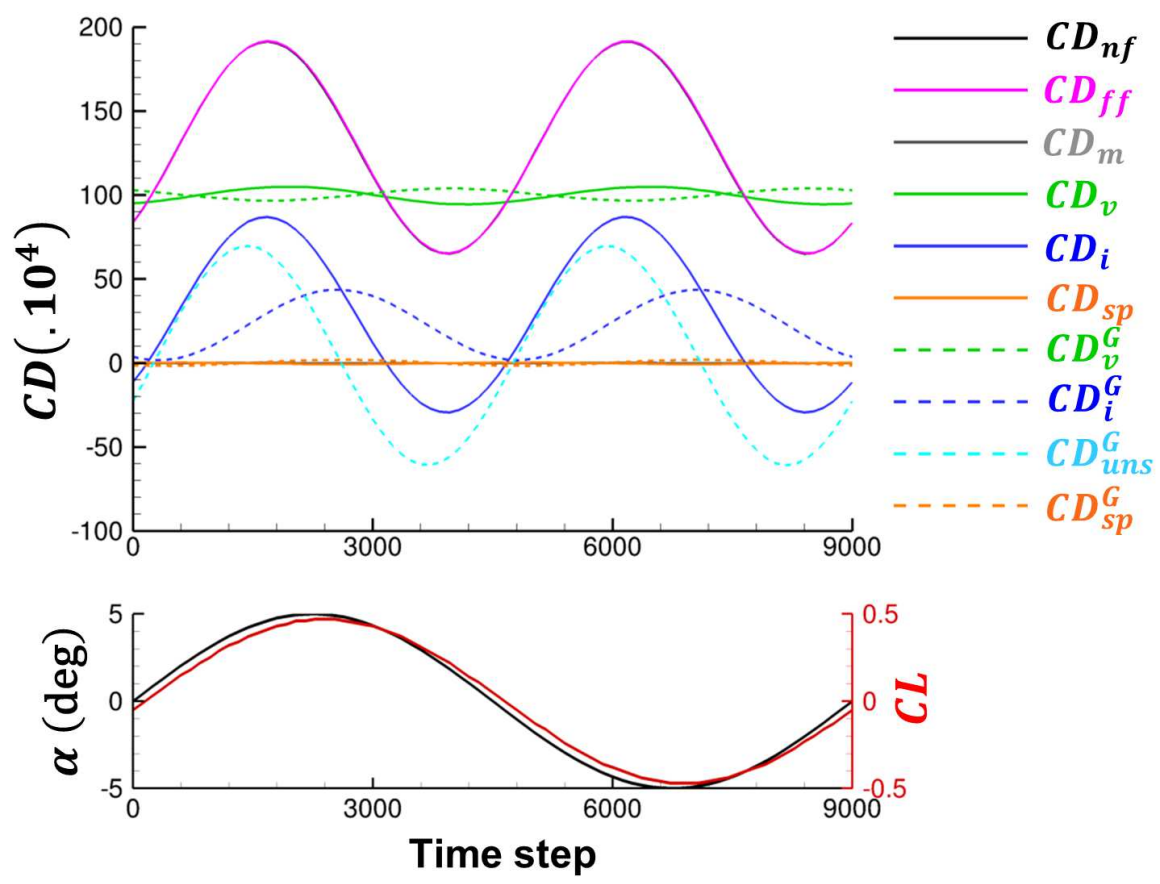

Figure 14. Evolution of the drag coefficients with respect to time over one period. 
of 85. The induced drag in dashed blue is not zero but remains positive. It is strongest when the angle of attack is the highest. Gariépy did not mention any result for the induced drag in his article. The unsteady drag (light blue) varies between -60 and 70 drag counts instead of -15 and 110 . The phase is also shifted. The spurious drag (dashed orange) is small, at most 2 drag counts.

The conclusion of this test case is that the new formulation appears to be robust, gives results consistent with the physics of the flow and is accurate. The induced drag is however still difficult to grasp. It is planned to study the same case in Euler flow and also in a 3D configuration in order to better understand and validate this decomposition.

\section{Conclusion}

Van der Vooren far-field drag breakdown method has been extended to unsteady flows. The new formulation includes unsteady terms into each drag coefficient rather than gathering them in an unsteady drag coefficient. Since Van der Vooren definition of the axial velocity defect is not always defined, a new expression introduced by Méheut and used by Gariépy is described and compared in various steady configurations. The results show that it is not as reliable as Van der Vooren definition and should be used with caution.

A theoretical analysis of Gariépy's formulation also reveals discrepancies. Both unsteady formulations were tested on three URANS configurations: a $2 \mathrm{D}$ buffeting case, a $2 \mathrm{D}$ vortex shedding case and a $2 \mathrm{D}$ oscillating body. The applications show that although we still lack understanding of the unsteady induced drag, the suggested decomposition gives good results. We get little spurious drag and modifications in the integration domains do not alter or put the coefficients out of phase.

In order to validate the breakdown between viscous and induced drag, oscillating cases are under study in Euler flows and on 3D configurations. Further work will deal with more complex URANS and DES test cases. A CROR test case should be the following application, aiming to quantify the true efficiency of such breakthrough engines. Vorticity-based formulations will also be investigated, since they are likely better suited to unsteady flows and induced drag, although they do not yet permit to split the drag into physical components.

\section{Acknowledgments}

The authors thank their colleagues from the Applied Aerodynamics Department M. Costes for his expertise on vorticity and moving bodies, D. Destarac for his support and great experience in far-field drag extraction.

\section{References}

${ }^{1}$ Betz, A., "A Method for the Direct Determination of Wing-Section Drag (in German)," Tech. rep., ZFM, 1925.

${ }^{2}$ Noca, F., On the Evaluation of Time-Dependent Fluid-Dynamic Forces on Bluff Bodies, Ph.D. thesis, California Institute of Technology, 1997.

${ }^{3}$ Wu, J.-Z., Ma, H.-Y., and Zhou, M.-D., Vorticity and Vortex Dynamics, Springer, 2006.

${ }^{4}$ Marongiu, C. and Tognaccini, R., "Far-Field Analysis of the Aerodynamic Force by Lamb Vector Integrals," AIAA Journal, Vol. 48, No. 11, November 2010, pp. 2543-2555.

${ }^{5} \mathrm{Xu}$, C.-Y., Chen, L.-W., and Lu, X.-Y., "Large-Eddy Simulation of the Compressible Flow past a Wavy Cylinder," Journal of Fluid Mechanics, Vol. 664, 2010, pp. 238-273.

${ }^{6}$ Gariépy, M., Trépanier, J.-Y., and Malouin, B., "Generalization of the Far-Field Drag Decomposition Method to Unsteady Flows," AIA A Journal, Vol. 51, No. 6, June 2013, pp. 1309-1319.

${ }^{7}$ Van der Vooren, J. and Destarac, D., "Drag/Thrust Analysis of a Jet-Propelled Transonic Transport Aircraft: Definition of Physical Drag Components," Aerospace Science and Technology, Vol. 8, 2004, pp. 545-556.

${ }^{8}$ Gariépy, M. and Trépanier, J.-Y., "A New Far Field Drag Decomposition Formulation Relevant to Highly Separated Flows," CASI AERO Conference, Montréal, Canada, 2011.

${ }^{9}$ Méheut, M. and Bailly, D., "Drag-Breakdown Methods from Wake Measurements," AIAA Journal, Vol. 46, No. 4, 2008, pp. $847-862$.

${ }^{10}$ Cambier, L. and Gazaix, M., "elsA: an Efficient Object-Oriented Solution to CFD Complexity," AIAA Paper, Vol. 2002, No. 0108, 2002.

${ }^{11}$ Mesquita, A.-L.-A., "Experimental Analysis or Airfoil for High Angle of Attack," Revista Virtual de Iniciao Acadmica da UFPA, Vol. 1, No. 2, jul 2001. 